Check for updates

Cite this: RSC Adv., 2018, 8, 7735

Received 29th November 2017 Accepted 12th February 2018

DOI: $10.1039 / c 7 r a 12898 c$

rsc.li/rsc-advances

\section{A carbonised sieve-like corn straw cellulose- graphene oxide composite for organophosphorus pesticide removal $\uparrow$}

\author{
Fengyue Suo, ${ }^{a}$ Guixian Xie, ${ }^{b}$ Jie Zhang, ${ }^{c}$ Jingyu Li, ${ }^{c}$ Changsheng $\mathrm{Li}^{\mathrm{c}}{ }^{\mathrm{C}}$ Xue Liu, ${ }^{\mathrm{C}}$ \\ Yunpeng Zhang, ${ }^{c}$ Yongqiang $\mathrm{Ma}^{\mathrm{C}}$ and MingShan $\mathrm{Ji}$ (iD *a
}

\begin{abstract}
The development of efficient adsorbents for the removal of organophosphorus pesticides from water is a major challenge. In this work, we prepared an activated carbon derived from sieve-like cellulose/ graphene oxide composites (ACCE/G) for the removal of several organophosphorus pesticides. We employed corn straw to produce a sieve-like cellulose-graphene oxide composite (CCE/G); then, by treating CCE/G with potassium hydroxide at high temperatures, the efficient adsorbent ACCE/G was prepared. The adsorption capacity of ACCE/G is higher than those of other sorbents, including a multiwall carbon nanotube, graphitised carbon black, activated carbon, C18, and primary secondary amine adsorbent. The ACCE/G structure has been fully characterised via scanning electron microscopy, Fourier-transform infrared spectroscopy, X-ray photoelectron spectroscopy, X-ray diffraction and Brunauer-Emmett-Teller analysis. The maximum adsorption capacity of ACCE/G is $152.5 \mathrm{mg} \mathrm{g}^{-1}$ for chlorpyrifos. The mechanism, the thermodynamic properties, and the kinetics of the adsorption process have been investigated as well. Our findings demonstrate that the adsorption mechanism depends on the electron-donating abilities of the $\mathrm{S}$ and $\mathrm{P}$ atoms. Moreover, the Langmuir model gives the best fit for the isotherm data, and the adsorption efficiency of the ACCE/G is still over $80 \%$ after eight times of recycling, making ACCE/G a valuable candidate for the removal of OPPs.
\end{abstract}

\section{Introduction}

Organophosphorus pesticides (OPPs) are one of the most extensively used compounds to protect crops. ${ }^{1}$ However, the abuse of highly-toxic compounds leads to the pollution of the environment and of the crop and poultry products, eventually causing food safety issues. ${ }^{2}$ Several methods have emerged as promising techniques for the removal of OPPs, such as advanced oxidation, enzymatic biodegradation, and adsorption techniques. $^{3-6}$ Compared to the other methods mentioned above, adsorption has the advantages of low cost, easy to operate, and wide range of applications. Therefore, it is important to develop an efficient adsorbent for the removal of OPPs.

Corn straw is the typical source of cellulose because of its large-scale output across the world. ${ }^{7}$ The annual production of corn straw is $\sim 350$ million tons in China. In corn straw, the

${ }^{a}$ College of Plant Protection, Shenyang Agricultural University, Shenyang 110866, China

${ }^{b}$ College of Resources and Environment, Hunan Agricultural University, Changsha 410128, China

${ }^{c}$ College of Science, China Agricultural University, Beijing 100193, China

$\dagger$ Electronic supplementary information (ESI) available. See DOI: $10.1039 / \mathrm{c} 7 \mathrm{ra} 12898 \mathrm{c}$ content of cellulose is in the range of $28-36 \%$. Nowadays, most of the corn straw has been either employed to improve the soil fertility or burnt contributing to pollution. ${ }^{8}$ The cellulose, derived from natural sources, shows adsorption properties in various forms, including raw cellulose, modified cellulose, and as a precursor to activated carbon (AC). These distinct forms of cellulose can be used to remove pollutants, such as dyes, heavy metals, pesticides, and antibiotics. ${ }^{9-12}$ Recently, the use of AC derived from cellulose has been widely explored because of its high adsorption properties. Laszlo et al. prepared porous carbon utilising packing material as a source of cellulose to remove phenol from aqueous solution. ${ }^{\mathbf{1 3}}$ However, further systematic effort on the development of methods to efficiently derive AC from cellulose precursors is required. Moreover, the adsorption mechanisms for the removal of pollutants from wastewater also need to be investigated in more detail. Indeed, few studies have been published to explain the different adsorption mechanisms, such as the high surface area ${ }^{\mathbf{1 4}}$ and the effect of functional groups within adsorbents. Huang et al. synthesised an adsorbent, based on a modified ligno-cellulosic material, which can remove cadmium and lead from aqueous solutions. They found that the sorption was a chemical process involving surface chelation and ion exchange. ${ }^{15}$

Graphene oxide (GO), the precursor of graphene, is a twodimensional (2D) carbon sheet with oxygen-containing 
functional groups. ${ }^{16}$ Due to its conductivity, GO can easily be combined with polymers and other materials, forming composite materials with enhanced properties, such tensile strength, elasticity, and conductivity. ${ }^{17}$ According to previous studies, GO and its derivatives can also work as absorbents for the removal of antibiotics, metal ions, dyes, and pesticides. ${ }^{\mathbf{1 8 - 2 1}}$

Herein, we report on the synthesis of AC which is derived from a novel sieve-like cellulose-graphene oxide composite. The cellulose was extracted from corn straw which is biodegradable, biocompatible, and economical. The so-synthesised composite material was successfully used as an adsorbent to remove OPPs from contaminated water. The physical-chemical characteristics of the so-prepared composite had been assessed as well. Finally, the adsorption performance and the mechanisms for the removal of OPPs from the water were also discussed systematically.

\section{Experimental methods and materials}

\subsection{Materials}

Corn straw was supplied by a local farm of Northeast China. After cut into small pieces (length $2-3 \mathrm{~cm}$ ), the corn straw was washed with deionised water and then dried at $60{ }^{\circ} \mathrm{C}$ for $24 \mathrm{~h}$. The dried material was then ground into powder. Dichlorvos, dimethoate, chlorpyrifos, chlorfenvinphos, methidathion, and profenofos were obtained from the Institute of the Control of Agrochemicals, Ministry of Agriculture, China. Chromatographic grade ethyl acetate was purchased from Mreda Co., Ltd. (Beijing, China). Graphite was obtained from Qingdao Hensen Graphite Co., Ltd. (Qingdao, China). Activated carbon (surface area/1000 $\mathrm{m}^{2} \mathrm{~g}^{-1}$ ) was obtained by Sinopharm Chemical Reagent Co., Ltd. (Shanghai, China), whereas primary secondary amine adsorbent (PSA, surface area/480 $\mathrm{m}^{2} \mathrm{~g}^{-1}$ ), graphitised carbon black (GCB, surface area/200 $\mathrm{m}^{2} \mathrm{~g}^{-1}$ ), and C18 (surface area/600 $\mathrm{m}^{2} \mathrm{~g}^{-1}$ ) were obtained from Bonna-Agela China (Tianjin, China).

\subsection{Preparation of the graphene oxide}

GO powder used in this work was obtained according to modified Hummers' method. ${ }^{22}$ Detailed experimental steps can be referred to our previous works. ${ }^{23}$

\subsection{Preparation of the cellulose from corn straw}

Cellulose was extracted from corn straw with modified Kurschner-Hoffer method. ${ }^{24} 20 \mathrm{~g}$ of corn straw powder was

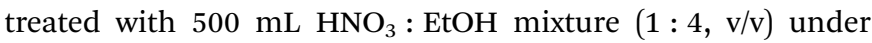
reflux and stirred at $100{ }^{\circ} \mathrm{C}$ until the powder was turned into white. The product was washed with deionised water and then was dried at $60{ }^{\circ} \mathrm{C}$ for $12 \mathrm{~h}$.

\subsection{Synthesis of the AC derived from cellulose-graphene oxide composite (ACCE/G)}

According to the method used by Zheng et al. for the preparation of a porous graphene/activated carbon composite, ${ }^{25} 0.05 \mathrm{~g}$ of GO powder was first added to $50 \mathrm{~mL}$ of deionised water and then dispersed by ultrasonication with a power of $100 \mathrm{~W}$ for $30 \mathrm{~min}$. After that, $1.5 \mathrm{~g}$ of cellulose and $2.0 \mathrm{~g}$ of urea were added. After ultrasonication for other $30 \mathrm{~min}$, the mixture was transferred into Teflon autoclave and hydrothermally treated at $180^{\circ} \mathrm{C}$ for $24 \mathrm{~h}$. After filtering and washing with deionised water and ethanol, the obtained solid sample was dried at $60^{\circ} \mathrm{C}$ under vacuum for $12 \mathrm{~h}$ to obtain the intermediate product: carbonised cellulose-graphene oxide composite (CCE/G). The CCE/G was mixed with $\mathrm{KOH}$ at different weight ratios $(0,1 / 4,1 / 2,4 / 1)$ and was heat treated $\left(400{ }^{\circ} \mathrm{C}, 500{ }^{\circ} \mathrm{C}, 600{ }^{\circ} \mathrm{C}\right)$ for $1 \mathrm{~h}, 2 \mathrm{~h}, 3 \mathrm{~h}$ in a crucible introduced into a muffle furnace connected to the outside atmosphere. After washed with $1 \mathrm{~mol} \mathrm{~L}^{-1} \mathrm{HCl}$ solution and deionised water, the obtained product was dried at $60^{\circ} \mathrm{C}$ for $12 \mathrm{~h}$ to obtain the activated carbonised cellulose-graphene oxide composite (ACCE/G). The AC produced from corn stalk cellulose without incorporation of graphene oxide (ACCE) was also prepared.

\subsection{Characterisation}

The samples were characterised via scanning electron microscopy (SEM, Quanta FEG 250), transmission electron microscopy (TEM, Tecnai G2 F20), diffuse reflectance infrared Fourier transform spectroscopy (DRIFT, Spectrum 100 FT-IR), and Raman spectroscopy (Renishaw RM2000). The crystallinity of the sample was investigated by X-ray diffraction (XRD, BRUKER D8 ADVANCE), whereas the Brunauer-Emmett-Teller (BET) surface area (Micromeritics ASAP2020), pore volume, and pore size were characterised using nitrogen adsorption at liquid nitrogen temperatures, and via X-ray photoelectron spectroscopy (XPS, Thermo SCIENTIFIC ESCALAB 250Xi).

\subsection{Adsorption experiments}

Different ACCE/G doses (5 to $120 \mathrm{mg}$ ) were investigated to determine the proper doses for removing the OPPs. To test the effect of time on adsorption, $50 \mathrm{mg}$ ACCE/G was added to the freshly prepared solution of OPPs $\left(10 \mathrm{~mL}, 2 \mathrm{mg} \mathrm{\textrm {L } ^ { - 1 }}\right)$. The samples were stirred on a vortex mixer for 0 (shaken five times by hand), 0.5, 1, 1.5, 2, 2.5, 3 and $5 \mathrm{~min}$. The pHs of the solutions containing OPPs (1-11) were also examined $(10 \mathrm{~mL}$, $\left.2 \mathrm{mg} \mathrm{L}^{-1}\right)$. The kinetic study was carried out in several $10 \mathrm{~mL}$ centrifuge tubes containing $5 \mathrm{~mL}$ of chlorpyrifos solution $\left(2 \mathrm{mg} \mathrm{L}^{-1}\right.$ ). The centrifuge tubes were sealed and placed in an oscillator at a speed of $170 \mathrm{rpm}$ with a temperature of $298 \mathrm{~K}$ for different durations ( $1 \mathrm{~min}, 3 \mathrm{~min}, 5 \mathrm{~min}, 7 \mathrm{~min}, 10 \mathrm{~min}, 20 \mathrm{~min}$, $30 \mathrm{~min}, 60 \mathrm{~min}, 120 \mathrm{~min}, 180 \mathrm{~min}, 300 \mathrm{~min}$ ). To obtain the adsorption isotherm, $5 \mathrm{mg}$ ACCE/G was added to the chlorpyrifos solution $(5 \mathrm{~mL})$ with a concentration ranging from 1 to $120 \mathrm{mg} \mathrm{L}^{-1}$. Then, the centrifuge tubes were sealed and placed in an oscillator at a speed of $170 \mathrm{rpm}$ with a temperature of 298 $\mathrm{K}, 308 \mathrm{~K}$, and $318 \mathrm{~K}$, until equilibrium was reached.

After the adsorption process, the solution was centrifuged for $5 \mathrm{~min}$ at $3800 \mathrm{rpm}$; the supernatant was discarded. Ethyl acetate $(10 \mathrm{~mL})$, used as a desorption solvent, and $\mathrm{NaCl}(1 \mathrm{~g})$ were added, and the mixture was vortexed for $3 \mathrm{~min}$ to desorb the analytes. Then the mixture was centrifuged at $3800 \mathrm{rpm}$ for 
$5 \mathrm{~min} .1 \mathrm{~mL}$ of the supernatant was dehydrated by anhydrous $\mathrm{MgSO}_{4}(50 \mathrm{mg}$ ), after vortexed for $1 \mathrm{~min}$ and centrifuged at $10000 \mathrm{rpm}$ for $1 \mathrm{~min}$. The concentrations of the OPPs in the supernatant were determined by the gas chromatography with flame photometric detection (GC-FPD). The temperature of the injector was held at $220{ }^{\circ} \mathrm{C}$ in a splitless mode. The oven temperature was programmed as follows: it was initially held for $1 \mathrm{~min}$ at $80{ }^{\circ} \mathrm{C}$, then increased to $180{ }^{\circ} \mathrm{C}$ at the rate of $25{ }^{\circ} \mathrm{C} \min ^{-1}$, and further increased to $260{ }^{\circ} \mathrm{C}$ at the rate of $15{ }^{\circ} \mathrm{C} \min ^{-1}$. The temperature of the detector was set at $260{ }^{\circ} \mathrm{C}$. The injection volume was $1 \mathrm{~mL}$. Ultrapure nitrogen was used as

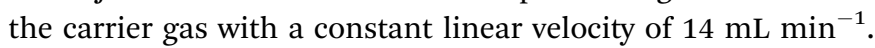
The flow rates of hydrogen and no air were $80 \mathrm{~mL} \mathrm{~min}{ }^{-1}$ and $120 \mathrm{~mL} \mathrm{~min}^{-1}$, respectively.

\section{Results and discussion}

\subsection{Morphology and structures of CCE/G}

SEM technique has been utilised to observe the surface morphology of the products. The top view of CCE/G (Fig. 1a) displays a layer with ordered macrospores. Pores can also be observed on the surface of cellulose in Fig. S1a and b. $\dagger$ Munro et al. found that nitric acid could cause "surface cracking" on cellulose. ${ }^{26}$ After the dehydration polymerisation in the process of hydrothermal carbonisation, the size of the pores increases, and the sieve-like structure is formed (Fig. S1c $\dagger$ ). Cellulose has a larger particle size than GO (Fig. S1b and $d \dagger$ ). The surface of CCE (prepared without the presence of GO) is clean and smooth (Fig. S1 $\dagger$ ), whereas CCE/G (prepared in the presence of GO) has the sieve-like structure similar to that of CCE. GO nanoparticles are tightly attached to the surface of cellulose (Fig. 1a and d). After activation by $\mathrm{KOH}$, the particle size of ACCE is smaller than that of ACCE/G (Fig. 1b and c), as further confirmed via XPS. Both the high-resolution images of ACCE and ACCE/G exhibit a porous structure (Fig. 1e and f). However, the image of ACCE/G shows wrinkled nanosheet morphology. TEM images of ACCE/G (Fig. S1c $\dagger$ ) further confirm that the presence of GO promotes the formation of porous nanosheets.

We have conducted FT-IR experiments to provide insight into the structures of CCE, CCE/G, ACCE, and ACCE/G. The FT-

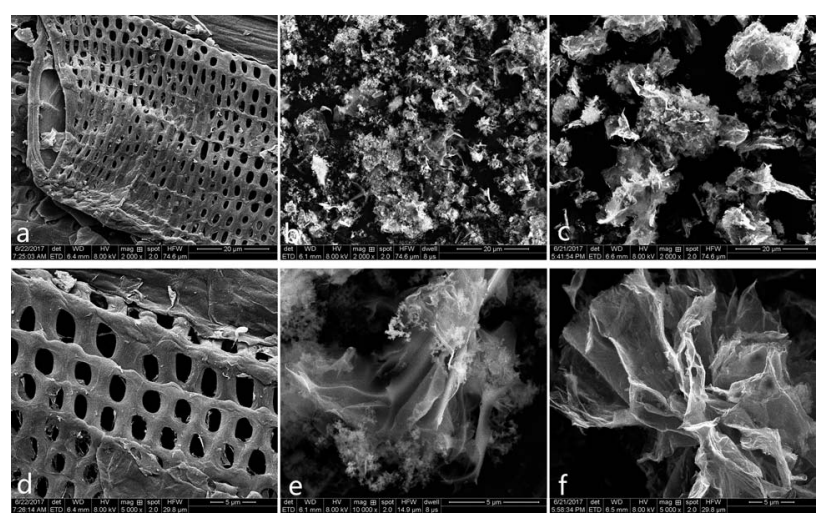

Fig. 1 SEM images of CCE/G (a) and (d); SEM images of ACCE (b) and (e); SEM images of ACCE/G (c) and (f).
IR spectra show that the absorbance peaks at $3382 \mathrm{~cm}^{-1}$, $2897 \mathrm{~cm}^{-1}, 1370 \mathrm{~cm}^{-1}, 1319 \mathrm{~cm}^{-1}, 1164 \mathrm{~cm}^{-1}, 1112 \mathrm{~cm}^{-1}$, $1060 \mathrm{~cm}^{-1}$, and $897 \mathrm{~cm}^{-1}$ are associated with the cellulose (Fig. 2b). The structures of cellulose, HTC cellulose (CCE) and HTC prepared in the presence of GO (CCE/G) are, indeed, similar (Fig. 2), which can be further confirmed via XRD (Fig. S2 $\dagger$ ). However, the absorption bands of CCE/G are weaker and broader than those of cellulose, suggesting that dehydration and aromatisation reactions take place to a higher extent in this case than during the hydrothermal carbonisation. ${ }^{\mathbf{1 4}}$ The absorbance peaks of $\mathrm{OH}$ groups shift from $3382 \mathrm{~cm}^{-1}$ to $3261 \mathrm{~cm}^{-1}$, whereas the absorbance peak associated to the vibration mode of the $\mathrm{C}-\mathrm{O}-\mathrm{C}$ pyranose-ring skeletal moves from $1060 \mathrm{~cm}^{-1}$ for CCE to $1052 \mathrm{~cm}^{-1}$ for CCE/G. ${ }^{27,28}$ This phenomenon may indicate the presence of an interaction between the carboxy groups of GO and the oxygen groups in the cellulose. The treatment with $\mathrm{KOH}$ modifies the spectra of the composite materials. The peaks of ACCE and ACCE/G are weaker and broader than those of CCE and CCE/G. The heat treatment with $\mathrm{KOH}$ leads to a graphitic/aromatic structure with fewer oxygencontaining groups. This phenomenon is further confirmed via XPS: the peaks of ACCE/G are weaker than those of ACCE, suggesting that the presence of GO could enhance the level of aromatisation.

X-ray diffraction experiments have been performed to explore the crystallographic structures of the composite materials as well. In the Fig. S2a, $\dagger$ according to the characteristic diffraction peaks of cellulose ${ }^{29}$ the spectra of CCE and CCE/G exhibit two main peaks at $2 \theta=16.18^{\circ}$ and $22.64^{\circ}$, which correspond to the (110) and (200) planes. This suggests that cellulose, CCE, and CCE/G have the same crystallographic structure. However, the intensity of these two peaks increases slightly passing from CCE to CCE/G due to the presence of GO, which may lead to a higher polymerisation degree.$^{30}$ Because of the volatile reducing gases produced during hydrothermal carbonisation, the presence of urea can restore p-conjugation structure and promote the removal of oxygen-functional groups. ${ }^{31}$ The treatment with $\mathrm{KOH}$ has influenced the X-ray diffraction spectra of ACCE and ACCE/G. The peaks of ACCE

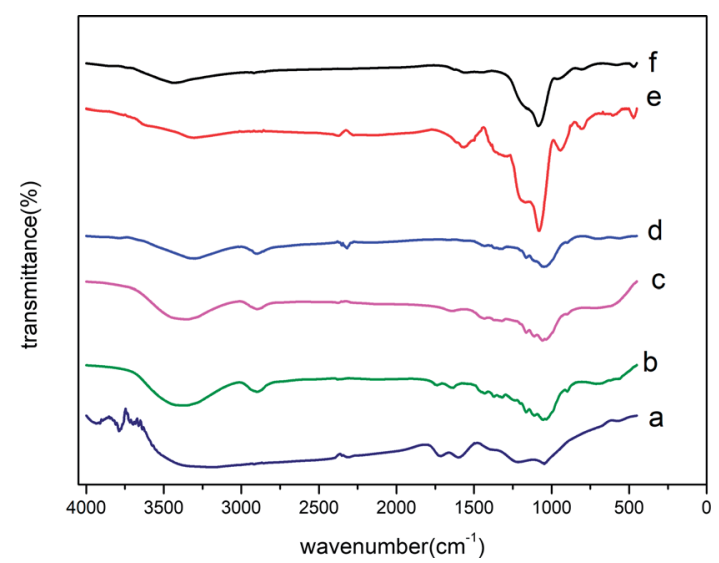

Fig. 2 FT-IR spectra of GO (a), cellulose (b), CCE (c), CCE/G (d), ACCE (e), and ACCE/G (f). 

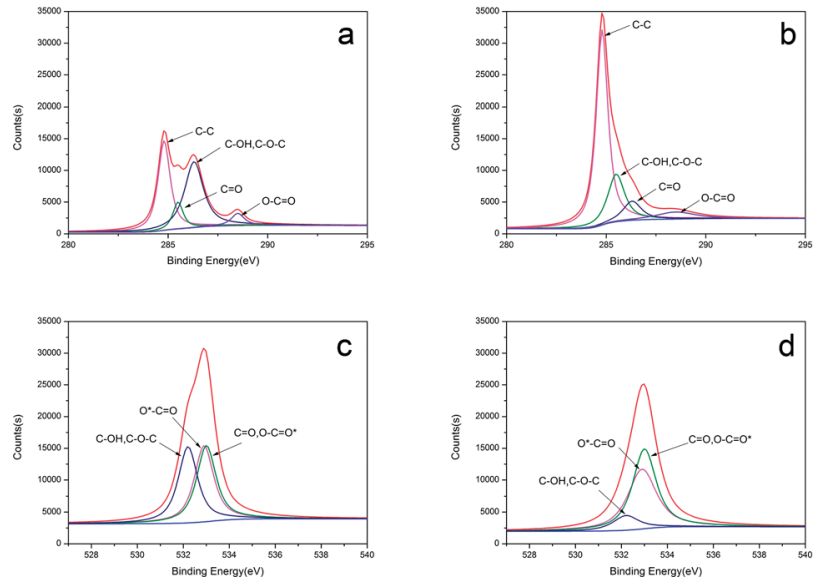

Fig. 3 XPS images of CCE/G C 1s (a), ACCE/G C 1s (b), CCE/G O 1s (c), and $A C C E / G O$ is (d).

and ACCE/G become weaker and broader, probably suggesting that a drastic morphological transformation takes place during the activation process $^{32}$ and that the particle size of the composite has been reduced during the chemical activation process. ${ }^{25}$ The peak of ACCE was broader than that of ACCE/G, which indicates that the particle size of ACCE was smaller than ACCE/G.

Raman spectroscopy has been applied to investigate the carbon structures of CCE/G, ACCE, and ACCE/G. Fig. S2b $\dagger$ illustrates the Raman patterns of the CCE/G, ACCE, and ACCE/ G. The D and $\mathrm{G}$ peaks at $1352 \mathrm{~cm}^{-1}$ and $1598 \mathrm{~cm}^{-1}$, can be observed for the three composite materials. The intensity of the $D$ band at $1352 \mathrm{~cm}^{-1}$ is attributed to the presence of condensed benzene rings and/or defected graphitic structures. Therefore, the presence of the D band in the Raman spectra of the ACCE and ACCE/G confirms the formation of small aromatic clusters during chemical activation. The $\mathrm{G}$ band at $1598 \mathrm{~cm}^{-1}$ originates from the $\mathrm{sp}^{2}$ hybridisation of $\mathrm{C}-\mathrm{C}$ bonds. ${ }^{33}$ The ratio of the intensity between D band and G band for ACCE and ACCE/G increases from 0.84 to 0.98 ; this increased defect degree is apparently due to the increased ratio of $\mathrm{sp}^{3}$ hybridised carbon in the presence of GO. The broad band at $2680 \mathrm{~cm}^{-1}$ of ACCE/G and ACCE can be assigned to the 2D peak, which is an overtone

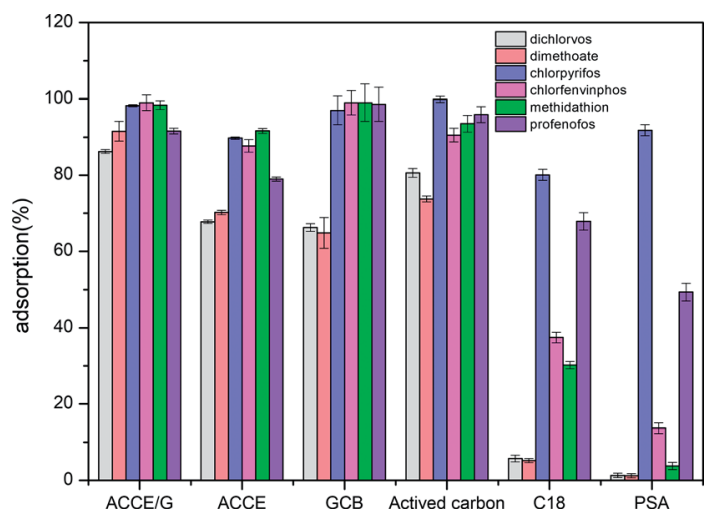

Fig. 4 Comparison of the adsorption efficiency of ACCE/G, GCB, AC, C18, PSA, and ACCE towards six OPPs.

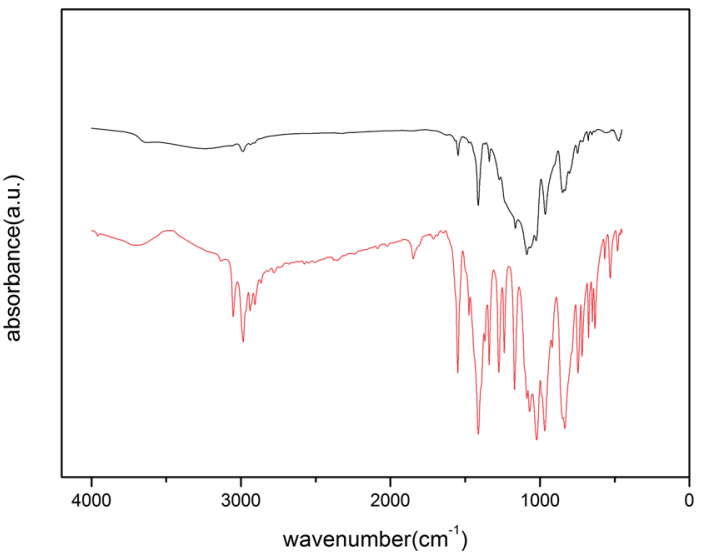

Fig. 5 IR spectra of chlorpyrifos (first line) and ACCE/G after adsorption of OPPs in water (second line).

of the D band. The appearance of a well-defined 2D band is known to be related to the development of a significant graphitic character in carbon materials. ${ }^{16}$

The component elements have been further analysed via surface sensitive high-resolution XPS to determine the composition of the materials before and after the $\mathrm{KOH}$ treatment. The C 1s binding energies are $284.2 \mathrm{eV}, 284.8 \mathrm{eV}, 285.5 \mathrm{eV}, 286.3 \mathrm{eV}$, and $288.5 \mathrm{eV}$ (Fig. 3a and b) for the following bonds: C-C, C$\mathrm{OH} / \mathrm{C}-\mathrm{O}-\mathrm{C}, \mathrm{C}=\mathrm{O}$, and $\mathrm{O}=\mathrm{C}-\mathrm{O} \cdot{ }^{34}$ Fig. $5 \mathrm{c}$ and d show the $\mathrm{O} 1 \mathrm{~s}$ spectrum can be fitted to three component peaks at $532.9 \mathrm{eV}$, $532.2 \mathrm{eV}, 533.0 \mathrm{eV}$, which represent $\mathrm{C}-\mathrm{O} / \mathrm{O}-\mathrm{C}-\mathrm{O}^{*}$ groups, $\mathrm{C}-\mathrm{OH}$ and/or C-O-C groups, ${ }^{*} \mathrm{O}-\mathrm{C}-\mathrm{O}$, respectively. ${ }^{35}$ After activation by $\mathrm{KOH}$, the content of carbon increases, while the content of oxygen decrease, which is in agreement with the FT-IR spectra.

Detailed information on the BET surface area and pore size distribution of the CCE/G and ACCE/G samples are summarised in Table 1 . The porosity has substantially increased during the heat treatment with $\mathrm{KOH}$. After the activation process, the BET of ACCE/G dramatically increases to $160 \mathrm{~m}^{2} \mathrm{~g}^{-1}$, which is almost 18 times that of CCE/G $\left(9.0160 \mathrm{~m}^{2} \mathrm{~g}^{-1}\right)$. Therefore, it is evident that the process of activation plays an essential role in the creation of porosity. ${ }^{36}$ The increase of the BET and the volume of microporous may be responsible for the OPPs adsorption.

\subsection{Optimisation of the ACCE/G}

After activated by $\mathrm{KOH}$, the adsorption capacity of the ACCE/G has been increased dramatically. The adsorption capacity of ACCE is not excellent towards dichlorvos and dimethoate when compared with that of the ACCE/G. Although the adsorption of the other four OPPs (chlorpyrifos, chlorfenvinphos,

Table 1 Textural parameters calculated from the $\mathrm{N}_{2}\left(-196{ }^{\circ} \mathrm{C}\right)$ adsorption isotherms for the materials used in this work

\begin{tabular}{lcll}
\hline Material & $S$ Bet $\left(\mathrm{m}^{2} \mathrm{~g}^{-1}\right)$ & $\begin{array}{l}\text { Volume total } \\
\left(\mathrm{cm}^{3} \mathrm{~g}^{-1}\right)\end{array}$ & $\begin{array}{l}\text { Diameter average } \\
(\mathrm{nm})\end{array}$ \\
\hline $\mathrm{CCE} / \mathrm{G}$ & 9.0137 & 0.014299 & 6.33296 \\
$\mathrm{ACCE} / \mathrm{G}$ & 160.3765 & 0.154315 & 3.84881
\end{tabular}


methidathion, profenofos) are more than $80 \%$, the ACCE/G has a better adsorption capacity than ACCE (Fig. S3a $\dagger$ ).

We have optimised the synthesis condition to obtain the most adsorbing ACCE/G. By modulating the temperature and the time of the HTC, we found that these two parameters have little effect on the adsorption ability of the ACCE/G. Therefore, we have varied the conditions of the process of activation by $\mathrm{KOH}$. After chemical activation $(\mathrm{HTC} / \mathrm{KOH}=1 / 4$, heat-treated $500{ }^{\circ} \mathrm{C}$ for $2 \mathrm{~h}$ ), the average adsorption efficiency of the composite has been increased dramatically. The best weight ratio is $\mathrm{HTC} / \mathrm{KOH}=1 / 4$ (Fig. $\mathrm{S} 3 \mathrm{~b} \dagger$ ). To determine the optimal temperature, the $\mathrm{CCE} / \mathrm{G}$ composite was heat-treated for 2 hours at $400{ }^{\circ} \mathrm{C}, 500{ }^{\circ} \mathrm{C}$, and $600{ }^{\circ} \mathrm{C}$ with $\mathrm{KOH}(\mathrm{HTC} / \mathrm{KOH}=1 / 4)$. However, when the temperature has reached $600{ }^{\circ} \mathrm{C}$, there is a little amount of product left. As shown in Fig. S3c, $\dagger$ the adsorption capacity of the composite is higher when the heattreating temperature is $500{ }^{\circ} \mathrm{C}$. After heat-treated for 2 hours and 3 hours, no significant difference can be observed in the adsorption capacity of the product (Fig. S3d†). For economic and environmental concerns, we have chosen the heattreatment of 2 hours as the standard treatment in this experiment.

\subsection{Factors affecting the adsorption performance}

In this study, the absorbent dose, the adsorption time, the desorption time, and the $\mathrm{pH}$ of the solution have been optimised to achieve maximum adsorption efficiency.

3.3.1 Effects of dosage on adsorptive capacity of ACCE/G. The dose of adsorbent plays a crucial role in the adsorption efficiency. Different ACCE/G doses in the range of 5-150 mg (5 mg, $10 \mathrm{mg}, 20 \mathrm{mg}, 30 \mathrm{mg}, 50 \mathrm{mg}, 70 \mathrm{mg}, 100 \mathrm{mg}, 120 \mathrm{mg}$, and $150 \mathrm{mg}$ ) have added to $10 \mathrm{~mL}$ solutions containing $2 \mathrm{mg} \mathrm{L}^{-1}$ of each of the six organophosphorus pesticides. We have observed that the adsorption capacity has increased with increasing the adsorbent dosage because more surface area is available for adsorption (Fig. S4a $\dagger$ ). However, the data of $50 \mathrm{mg}$ shows equally good adsorption performance as $70 \mathrm{mg}$ and $100 \mathrm{mg}$. Hence, $50 \mathrm{mg}$ of adsorbent was chosen as the standard treatment in this experiment for economic concerns.

3.3.2 Effects of time on adsorptive capacity of ACCE/G. The time and method of mixing are key factors affecting the adsorption efficiency as well. In this test, $50 \mathrm{mg}$ of ACCE/G has been added to the solution and mixed by vortexing for different durations ( $0 \mathrm{~min}, 0.5 \mathrm{~min}, 1 \mathrm{~min}, 1.5 \mathrm{~min}, 2 \mathrm{~min}, 2.5 \mathrm{~min}$, $3 \mathrm{~min}$, and $5 \mathrm{~min}$ ) before the solutions have been tested. Fig. S4b $\dagger$ illustrates that no stirring (i.e., $0 \mathrm{~min}$, the solution was just shaken five times by hand) produces almost the same effect as stirring for $2.5 \mathrm{~min}$, with the exception of dichlorvos and dimethoate. When the time of vortexing reaches $2.5 \mathrm{~min}$, the adsorption of all the six OPPs exceeds 90\%. Therefore, vortexing for $2.5 \mathrm{~min}$ was chosen as the standard treatment in this experiment.

3.3.3 Effects of $\mathbf{p H}$ on adsorptive capacity of ACCE/G. For water samples, $\mathrm{pH}$ is a key factor affecting the ionic states of the target analytes and may, therefore, influence the adsorption properties of the ACCE/G. The $\mathrm{pH}$ of the solution was adjusted to $1,3,5,7,9$, and 11 with $\mathrm{HCl}$ or $\mathrm{NaOH}$. The data in Fig. S4c $\dagger$ shows no significant variation in adsorption over the $\mathrm{pH}$ range studied ( $\mathrm{pH}=1-7)$. Hence, no $\mathrm{pH}$ adjustments were applied in the following tests.

\subsection{Comparison with six other sorbents}

Six sorbents, including ACCE/G, multi-wall carbon nanotube, GCB, AC, C18, PSA, ACCE, have been tested, and their adsorption performance has been compared. The tests have been performed under the optimal conditions. Fig. 4 presents the results, showing that the adsorptive capacities of the PSA and C18 are unsatisfactory. The adsorption capacity of GCB, AC, and ACCE are not excellent for the cases of dichlorvos and dimethoate. Overall, the ACCE/G shows more performing adsorption capacity than the other five sorbents.

\subsection{Adsorption mechanism of ACCE/G for six OPPs}

The adsorption mechanism involving OPPs is complex since the OPPs contains several functional groups that would interact with the sorbent. According to our findings, the ACCE/G has a high surface area with a considerable amount of mesopores. Moreover, the strong $\pi$-bonding network in the OPPs and the electron-donating abilities of the $\mathrm{N}, \mathrm{S}$, and $\mathrm{O}$ atoms can also generally assist adsorption. In addition, the changes of ACCE/G after residual adsorption in IR (Fig. 5) shows that after the adsorption of chlorpyrifos, the $\mathrm{P}=\mathrm{S}$ peak has moved from $834 \mathrm{~cm}^{-1}$ to $845 \mathrm{~cm}^{-1}$, and the $\mathrm{P}-\mathrm{O}-\mathrm{C}$ of aromatic has shifted from $1701 \mathrm{~cm}^{-1}$ to $1163 \mathrm{~cm}^{-1}$, indicating that the $\mathrm{P}, \mathrm{S}$, and $\mathrm{O}$ atoms have promoted the adsorption. Although a study suggested that the adsorption mechanism was based on the strong $\pi$-bonding network of benzene rings ${ }^{23}$ the band corresponding to the pyridine-ring vibration has not been modified after the adsorption. The possible reason may be that the pyridine ring is an electron-deficient group; further, the atom $\mathrm{C} 1$ attached to the pyridine is an electron withdrawing group. This may occur due to the weakened $\pi$-bonding network of the pyridine ring. In summary, the electron-donating abilities of $\mathrm{P}$ and $\mathrm{S}$ atoms promote the adsorption.

3.5.1 Adsorption kinetics. The adsorption rate and adsorption equilibrium time play a key role in the adsorption mechanism. To further understand the kinetics of the adsorption process, the experimental kinetic adsorption data of chlorpyrifos have been introduced into two kinetic models: the pseudo-first-order model and the pseudo-second-order model. The Lagergren pseudo-first-order model is based on the assumption that the adsorption process has a rapid initial phase. ${ }^{37}$ The model is expressed by the following equation:

$$
\ln \left(Q_{\mathrm{e}}-Q_{t}\right)=\ln Q_{\mathrm{e}}-\frac{k_{1} t}{2.303}
$$

where $Q_{\mathrm{e}}$ and $Q_{t}$ are the amounts of chlorpyrifos $\left(\mathrm{mg} \mathrm{g}^{-1}\right)$ adsorbed on adsorbent at equilibrium and at a given time $t(\mathrm{~min})$, whereas $k_{1}$ is the rate constant of adsorption $\left(\mathrm{g} \mathrm{mg}^{-1} \mathrm{~min}^{-1}\right)$.

The experimental values $Q_{\mathrm{e}}$ are not in agreement with the calculated ones as obtained from the linear plots. Moreover, the values of the correlation coefficient $\left(R^{2}\right)$ are relatively low 
(Fig. S5a $\dagger$ ), indicating that the adsorption process does not comply with the pseudo-first-order rate model.

The pseudo-second-order rate model is based on the assumption that the adsorption is rate-controlling which can be expressed by the following equation: ${ }^{38}$

$$
\frac{t}{Q_{t}}=\frac{1}{K_{2} Q_{\mathrm{e}}{ }^{2}}+\frac{t}{Q_{\mathrm{e}}}
$$

where $K_{2}$ is the rate constant for the pseudo-second-order adsorption process.

The linear plots of $t / Q_{t}$ versus $t$ show a correlation between experimental and calculated values at different initial concentrations (Fig. $\mathrm{S} 5 \mathrm{~b} \dagger$ ). The $R^{2}$ value for the pseudo-second-order kinetic model (Fig. S6 $\dagger$ ) is much higher than the value associated to the pseudo-first-order kinetics model, suggesting that the experimental data fit the pseudo-second-order kinetic model $\left(R^{2}>0.99\right) .{ }^{39}$ Similar results were acquired by He et al. ${ }^{37}$ and Cheng et al. ${ }^{40}$ They used treated biofilms as biosorbents for the heavy metals removal.

3.5.2 Adsorption isotherms. In this paper, chlorpyrifos has been selected to derive the adsorption isotherms of ACCE/G. Fig. S5c $\dagger$ shows the adsorption isotherms of chlorpyrifos on ACCE/G over the concentration from 1 to $100 \mathrm{mg} \mathrm{L}^{-1}$ at $298 \mathrm{~K}$, $308 \mathrm{~K}$, and $318 \mathrm{~K}$. To understand the chlorpyrifos adsorption behaviours on the ACCE/G, two equilibrium models have been applied to evaluate the experimental data. The Langmuir model is based on the hypothesis that the absorption of metal ions occurs on a homogeneous surface through a single layer. However, the Freundlich model is founded on the adsorption on the heterogeneous surface reference. The two models can be described using eqn (3) and (4), respectively: ${ }^{\mathbf{4 1}}$

$$
\begin{aligned}
\frac{C_{\mathrm{e}}}{q_{\mathrm{e}}} & =\frac{1}{q_{\mathrm{m}} K_{\mathrm{L}}}+\frac{C_{\mathrm{e}}}{q_{\mathrm{m}}} \\
\ln q_{\mathrm{e}} & =\ln K_{\mathrm{F}}+\frac{\ln C_{\mathrm{e}}}{n}
\end{aligned}
$$

where $K_{\mathrm{L}}\left(\mathrm{L} \mathrm{mg}^{-1}\right)$ is the Langmuir equilibrium constant, $q_{\mathrm{m}}$ is the maximum adsorption capacity $\left(\mathrm{mg} \mathrm{g}^{-1}\right)$, and $K_{\mathrm{F}}\left(\left(\mathrm{mg} \mathrm{g}^{-1}\right)(\mathrm{L}\right.$ $\left.\mathrm{mg}^{-1}\right)^{1 / n}$ ) and $n$ are the Freundlich constants. The Freundlich constants are related to the adsorption capacity and adsorption intensity: with the increase the $K_{\mathrm{F}}$ and $n$ values, the adsorption capacity of adsorbent rises as well. ${ }^{42}$

For Langmuir isotherm model, the correlation coefficients $R^{2}$ are larger than 0.96 , which are higher than those for the Freundlich model (Table 2). The suitable fit of Langmuir model indicates that chlorpyrifos likely adsorbs forming monolayer coverage on the surface of ACCE/G. ${ }^{43}$ Dried activated sludge was used by Yang et al. as a biosorbent for zinc(II) removal, and also found that the adsorption takes place on a homogeneous surface by monolayer sorption as well. ${ }^{41}$ Furthermore, the maximum adsorption capacities and $K_{\mathrm{L}}$ for chlorpyrifos on the ACCE/G increases with increasing temperature, indicating that higher temperature is more favorable to the adsorption process.

3.5.3 Effect of the temperature on the adsorption. We have also conducted experiments $298 \mathrm{~K}, 308 \mathrm{~K}$, and $318 \mathrm{~K}$ to study the thermodynamics of the adsorption process of chlorpyrifos. The thermodynamic parameters can be calculated by the Van't Hoff equation: ${ }^{\mathbf{4}}$

$$
\begin{gathered}
\Delta G^{0}=-R T \ln K_{0} \\
\ln K_{0}=-\frac{\Delta G^{0}}{R T}=\frac{\Delta S}{R}-\frac{\Delta H^{0}}{R T}
\end{gathered}
$$

In eqn (5) and (6), $R$ is the gas constant $\left(8.314 \mathrm{~J} \mathrm{~mol}^{-1} \mathrm{~K}^{-1}\right) ; T$ is the sorption temperature in Kelvin; $K_{0}$ is the thermodynamic equilibrium constant, which is defined as follow:

$$
K_{0}=\frac{a_{\mathrm{s}}}{a_{\mathrm{e}}}=\frac{\gamma_{\mathrm{s}}}{\gamma_{\mathrm{e}}} \frac{q_{\mathrm{e}}}{C_{\mathrm{e}}}
$$

Here, $a_{\mathrm{s}}$ is the activity of the adsorbed chlorpyrifos, $a_{\mathrm{e}}$ is the activity of the chlorpyrifos in the solution at equilibrium, $\gamma_{\mathrm{s}}$ is the activity coefficient of the adsorbed chlorpyrifos, and $\gamma_{e}$ is the activity coefficient of chlorpyrifos in solution at equilibrium.

As the chlorpyrifos concentration in the solution decreases and approaches zero, the values of $K_{0}$ can be acquired by plotting $\ln \left(q_{\mathrm{e}} / C_{\mathrm{e}}\right)$ versus $q_{\mathrm{e}}$ and by extrapolating $q_{\mathrm{e}}$ to zero. Moreover, $\Delta G_{0}$ can be calculated using eqn (5); $\Delta H_{0}$ and $\Delta S_{0}$ can be obtained as the slope and the intercept of the eqn (6). ${ }^{39}$

Gibbs free energy values of the adsorption of chlorpyrifos on ACCE/G decrease with increasing temperature, indicating that the adsorption is a spontaneous and feasible process. The change of free energy for physical adsorption is in the range of -20 to $0 \mathrm{~kJ} \mathrm{~mol}^{-1}$, while chemical adsorption ranges from -400 to $-80 \mathrm{~kJ} \mathrm{~mol}^{-1} \cdot{ }^{45}$ Furthermore, the processes are endothermic since the enthalpy free energies values are. The positive value of $\Delta S_{0}$ indicates an increasing disorder at the solid/solution interface during the process of the adsorption of chlorpyrifos on ACCE/G (Table 3).

Table 2 Langmuir and Freundlich constants and correlation coefficients $\left(R^{2}\right)$ for chlorpyrifos adsorption on the ACCE/G

\begin{tabular}{lllllllll}
\hline & Langmuir & & & \multicolumn{2}{l}{ Freundlich } \\
\cline { 2 - 3 }$T(\mathrm{~K})$ & $Q_{\mathrm{m}}\left(\mathrm{mg} \mathrm{g}^{-1}\right)$ & $K_{\mathrm{L}}\left(\mathrm{L} \mathrm{mg}^{-1}\right)$ & $R^{2}$ & & $n$ & $K_{\mathrm{F}}$ & $R^{2}$ \\
\hline 298 & 120.48 & 0.8218 & 0.9842 & 1.2015 & 60.21 & 0.9768 \\
308 & 135.14 & 1.6817 & 0.9976 & & 1.4753 & 96.45 & 0.9799 \\
318 & 152.52 & 5.9998 & 0.9697 & & 2.6137 & 97.56 & 0.7983
\end{tabular}

Table 3 Thermodynamic parameters for the adsorption of chlorpyrifos on ACCE/G

\begin{tabular}{lllll}
\hline$T(\mathrm{~K})$ & $K_{0}$ & $\Delta G_{0}\left(\mathrm{~kJ} \mathrm{~mol}^{-1}\right)$ & $\Delta H_{0}\left(\mathrm{~kJ} \mathrm{~mol}^{-1}\right)$ & $\Delta S_{0}\left(\mathrm{~J} \mathrm{~mol}^{-1} \mathrm{~K}^{-1}\right)$ \\
\hline 298 & 4.6801 & -3.8237 & 19.7657 & 78.9946 \\
308 & 5.7083 & -4.4606 & & \\
318 & 7.7399 & -5.4104 & &
\end{tabular}




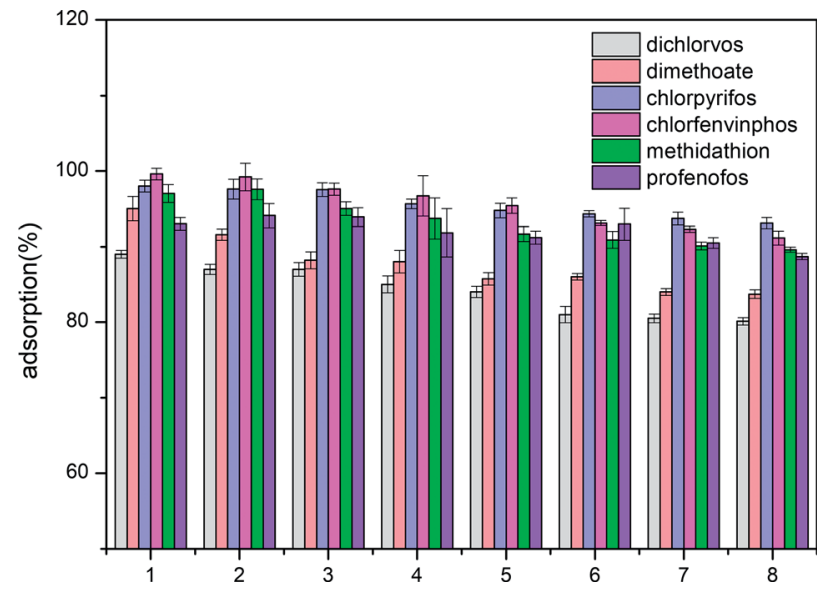

Fig. 6 Adsorption efficiency of organophosphorus pesticides by ACCE/G after various cycles of regeneration.

\subsection{Regeneration and reuse of ACCE/G}

Desorption experiments for the ACCE/G have also been carried out. Ethyl acetate has been chosen as the desorption solvent. Since the percentage of desorption is equal or higher than $90 \%$, the ACCE/G could be recycled. The results of eight consecutive adsorption/desorption cycles are shown in Fig. 6. The adsorption efficiency of the ACCE/G for OPPs is still over $80 \%$ after the eighth cycle, proving that $\mathrm{ACCE} / \mathrm{G}$ is a stable and efficient adsorbent for OPPs.

\section{Conclusions}

The AC derived from sieve-like cellulose-graphene oxide composite has an excellent adsorption capacity towards OPPs. Indeed, our newly synthesised composite material performs better than other five common adsorbents (multi-wall carbon nanotube, GCB, AC, C18, and PSA) in the removal of OPPs from water. The adsorption mechanism is mostly dependent on the electron-donating abilities of the $\mathrm{S}$ and $\mathrm{P}$ atoms. Moreover, the adsorption efficiency of the ACCE/G is still over $80 \%$ after eight times of recycling. Therefore, this work provides new insight into the application of the AC derived from corn straw as an adsorbent of pesticides in water.

\section{Conflicts of interest}

There are no conflicts to declare.

\section{Acknowledgements}

This study was financially supported by The National Key Technology Support Program of China (2015BAK45B01), The National Key Research and Development Program of China (2016YFD0201203) and Municipal Innovative Training Program for College Students of Beijing (2017Bj093).

\section{Notes and references}

1 S. Meseguer-Lloret, S. Torres-Cartas, M. Catalá-Icardo, E. F. Simó-Alfonso and J. M. Herrero-Martínez, Extraction and preconcentration of organophosphorus pesticides in water by using a polymethacrylate-based sorbent modified with magnetic nanoparticles, Anal. Bioanal. Chem., 2017, 409, 3561-3571.

2 Q. F. Yang, J. Wang, W. F. Zhang, F. B. Liu, X. Y. Yue, Y. N. Liu, M. Yang, Z. H. Li and J. L. Wang, Interface engineering of metal organic framework on graphene oxide with enhanced adsorption capacity for organophosphorus pesticide, Chem. Eng. J., 2017, 313, 19-26.

3 L. L. Zheng, F. W. Pi, Y. F. Wang, H. Xu, Y. Z. Zhang and $\mathrm{X}$. L. Sun, Photocatalytic degradation of acephate, omethoate, and methyl parathion by $\mathrm{Fe}_{3} \mathrm{O}_{4} @ \mathrm{SiO}_{2} @ \mathrm{mTiO}_{2}$ nanomicrospheres, J. Hazard. Mater., 2016, 315, 11-22.

4 E. Guivarch, N. Oturan and A. M. Oturan, Removal of organophosphorus pesticides from water by electrogenerated Fenton's reagent, Environ. Chem. Lett., 2003, 1, 165-168.

5 R. X. Li, C. P. Yang, H. Chen, G. M. Zheng, G. L. Yu and J. Y. Guo, Removal of triazophos pesticide from wastewater with Fenton reagent, J. Hazard. Mater., 2009, 167, 1028-1032.

6 R. D. Richins, I. Kaneva, A. Mulchandani and W. Chen, Biodegradation of organophosphorus pesticides by surfaceexpressed organophosphorus hydrolase, Nat. Biotechnol., 1997, 15, 984-987.

7 H. Y. Zhang, L. J. Chen, J. B. Li, M. S. Lu and L. J. Han, Quantitative characterization of enzyme adsorption and hydrolytic performance for ultrafine grinding pretreated corn stover, Bioresour. Technol., 2017, 234, 23-32.

8 I. Bertrand, M. Prevot and B. Chabbert, Soil decomposition of wheat internodes of different maturity stages: relative impact of the soluble and structural fractions, Bioresour. Technol., 2009, 155-163.

9 W. Li, T. T. Li, G. T. Li, L. B. An, F. Li and Z. M. Zhang, Electrospun $\mathrm{H}_{4} \mathrm{SiW}_{12} \mathrm{O}_{40}$ /cellulose acetate composite nanofibrous membrane for photocatalytic degradation of tetracycline and methyl orange with different mechanism, Carbohydr. Polym., 2017, 168, 153-162.

10 S. Hokkanen, E. Repo, S. Lou and M. Sillanpää, Removal of $\operatorname{arsenic}(\mathrm{v})$ by magnetic nanoparticle activated microfibrillated cellulose, Chem. Eng. J., 2015, 260, 886-894.

11 C. Zhang, R. Z. Zhang, Y. Q. Ma, W. B. Guan, X. L. Wu, X. Liu, H. Li, Y. L. Du and C. P. Pan, Preparation of cellulose/ graphene composite and its applications for triazine pesticides adsorption from water, ACS Sustainable Chem. Eng., 2015, 3, 369-405.

12 S. Y. Jia, Z. Yang, W. B. Yang, T. T. Zhang, S. P. Zhang, X. Z. Yang, Y. Y. Dong, J. Q. Wu and Y. P. Wang, Removal of $\mathrm{Cu}(\mathrm{II})$ and tetracycline using an aromatic ringsfunctionalized chitosan-based flocculant: enhanced interaction between the flocculant and the antibiotic, Chem. Eng. J., 2016, 283, 495-503. 
13 K. Laszlo, A. Bota, L. G. Nagy and I. Cabasso, Porous carbon from polymer waste materials, Colloids Surf., A, 1999, 151, 311-320.

14 Y. P. Guo and D. A. Rockstraw, Physical and chemical properties of carbons synthesized from xylan, cellulose, and kraft lignin by $\mathrm{H}_{3} \mathrm{PO}_{4}$ activation, Carbon, 2006, 44, 1464-1475.

15 Y. Q. Huang, C. Y. Yang, Z. C. Sun, G. M. Zeng and H. j. He, Removal of cadmium and lead from aqueous solutions using nitrilotriacetic acid anhydride modified lignocellulosic material, RSC Adv., 2015, 5, 11475-11484.

16 F. J. Martín-Jimeno, F. Suárez-García, J. I. Paredes, A. Martínez-Alonso and J. M. D. Tascón, Activated carbon xerogels with a cellular morphology derived from hydrothermally carbonized glucose-graphene oxide hybrids and their performance towards $\mathrm{CO}_{2}$ and dye adsorption, Carbon, 2015, 81, 137-147.

17 T. S. Anirudhan and J. R. Deepa, Nano-zinc oxide incorporated graphene oxide/nanocellulose composite for the adsorption and photo catalytic degradation of ciprofloxacin hydrochloride from aqueous solutions, $J$. Colloid Interface Sci., 2017, 490, 343-356.

18 Y. Gao, Y. Li, L. Zhang, H. Huang, J. Hu, S. M. Shah and $\mathrm{X}$. Su, Adsorption and removal of tetracycline antibiotics from aqueous solution by graphene oxide, J. Colloid Interface Sci., 2012, 368, 540-546.

19 H. H. Najafabadi, M. Irani, L. R. Rad, A. H. Haratameh and I. Haririan, Removal of $\mathrm{Cu}^{2+}, \mathrm{Pb}^{2+}$ and $\mathrm{Cr}^{6+}$ from aqueous solutions using a chitosan/graphene oxide composite nanofibrous adsorbent, Anal. Bioanal. Chem., 2015, 5, 16532-16539.

20 S. T. Yang, S. Chen, Y. L. Chang, A. Cao, Y. F. Liu and H. F. Wang, Removal of methylene blue from aqueous solution by graphene oxide, J. Colloid Interface Sci., 2011, 359, 24-29.

21 Q. F. Yang, J. Wang, W. T. Zhang, F. B. Liu, X. Y. Yue, Y. G. Liu, M. Yang, Z. H. H. Li and J. L. Wang, Interface engineering of metal organic framework on graphene oxide with enhanced adsorption capacity for organophosphorus pesticide, Chem. Eng. J., 2017, 313, 19-26.

22 R. E. Offeman and W. S. Hummers, Preparation of graphitic oxide, J. Am. Chem. Soc., 1958, 80, 1339.

23 X. T. Liu, H. Y. Zhang, Y. Q. Ma, X. L. Wu, L. X. Meng, Y. L. Guo, G. Yu and Y. Q. Liu, Graphene-coated silica as a highly efficient sorbent for residual organophosphorus pesticides in water, J. Mater. Chem. A, 2013, 1, 1875-1884.

24 J. A. S. Mendes, S. O. Prozil, D. V. Evtuguin and L. P. C. Lopes, Towards comprehensive utilization of winemaking residues: Characterization of grape skins from red grape pomaces of variety Touriga Nacional, Ind. Crops Prod., 2013, 43, 25-32.

25 C. Zheng, X. F. Zhou, H. L. Cao, G. H. Wang and Z. P. Liu, Synthesis of porous graphene/activated carbon composite with high packing density and large specific surface area for supercapacitor electrode material, J. Power Sources, 2014, 258, 290-296.
26 H. S. Munro and R. D. Short, A Study of the Low-Temperature Nitration of Cellulose in Mixed Acids, J. Appl. Polym. Sci., 1990, 39, 539-551.

27 M. Golshan, M. Salami-Kalajahi, H. Roghani-Mamaqani and M. Mohammad, Poly(propylene imine)dendrimer-grafted nanocrystalline cellulose: doxorubicin loading and release behavior, Polymer., 2017, 117, 287-294.

28 P. Zhang, S. J. Dong, H. H. Ma, B. X. Zhang, Y. F. Wang and X. M. Hu, Fractionation of corn stover into cellulose, hemicellulose and lignin using a series of ionic liquids, Ind. Crops Prod., 2015, 76, 688-696.

29 M. Cheng, Z. Y. Qin, Y. Y. Chen, S. Hu, Z. C. Ren and M. F. Zhu, Efficient extraction of cellulose nanocrystals through hydrochloric acid hydrolysis catalyzed by inorganic chlorides under hydrothermal conditions, ACS Sustainable Chem. Eng., 2017, 5, 4656-4664.

30 F. Beladi, S. Saber-Samandari and S. Saber-Samandari, Cellular compatibility of nanocomposite scaffolds based on hydroxyapatite entrapped in cellulose network for bone repair, Mater. Sci. Eng., C, 2017, 75, 385-392.

31 Z. B. Lei, L. Lu and X. S. Zhao, The electrocapacitive properties of graphene oxide reduced by urea, Energy Environ. Sci., 2012, 5, 6391-6399.

32 M. Sevilla, A. B. Fuertesa and R. Mokaya, High density hydrogen storage in superactivated carbons from hydrothermally carbonized renewable organic materials, Energy Environ. Sci., 2011, 4, 1400-1410.

33 Y. Wang, A. P. Fu, X. H. Liu, Y. Q. Wang, Y. H. Li, P. Z. Guo, H. L. Li and X. S. Zhao, Porous carbon directed growth of carbon modified $\mathrm{MnO}_{2}$ porous spheres for pseudo capacitor applications, J. Alloys Compd., 2017, 717, 341-349.

34 C. U. Pittman Jr, W. Jiang, Z. R. Yue, S. Gardner, L. Wang, H. Toghiani and C. A. leon y leon, Surface properties of electrochemically oxidized carbon fibers, Carbon., 1999, 37, 1797-1807.

35 J. F. Ma, J. X. Xing, K. Wang, H. Y. Yang, B. H. Fei and X. E. Liu, Inspired by efficient cellulose-dissolving system: Facile one-pot synthesis of biomass-based hydrothermal magnetic carbonaceous materials, Carbohydr. Polym., 2017, 164, 127-135.

36 F. Gao, G. H. Shao, J. Y. Qu, S. Y. Lv, Y. Q. Li and M. B. Wu, Tailoring of porous and nitrogen-rich carbons derived from hydrochar for high-performance supercapacitor electrodes, Electrochim. Acta, 2015, 155, 201-208.

37 H. J. He, Z. H. Xiang, X. J. Chen, H. Chen, H. Huang, M. Wen and C. P. Yang, Biosorption of Cd(II) from synthetic wastewater using dry biofilms from biotrickling filters, Int. J. Environ. Sci. Technol., 2017, DOI: 10.1007/s13762-0171507-8.

38 H. C. Shi, W. S. Li, L. Zhong and C. J. Xu, Methylene blue adsorption from aqueous solution by magnetic cellulose/ graphene oxide composite: equilibrium, kinetics, and thermodynamics, Ind. Eng. Chem. Res., 2013, 53, 1108-1118.

39 H. Z. Li, Z. B. Sun, L. Zhang, Y. X. Tian, G. J. Cui and S. Q. Yan, A cost-effective porous carbon derived from pomelo peel for the removal of methyl orange from aqueous solution, Colloids Surf., A, 2016, 489, 191-199. 
40 Y. Cheng, C. P. Yang, H. J. He and G. M. Zeng, Biosorption of $\mathrm{Pb}$ (II) Ions from Aqueous Solutions by Waste Biomass from Biotrickling Filters: Kinetics, Isotherms, and Thermodynamics, J. Environ. Eng., 2016, DOI: 10.1061/ (ASCE)EE.1943-7870.0000956.

41 C. P. Yang, J. Q. Wang, M. Lei, G. X. Xie, G. M. Zeng and S. L. Luo, Biosorption of zinc(II) from aqueous solution by dried activated sludge, J. Environ. Sci., 2010, 22(5), 675-680.

42 J. S. Zhang, S. J. Chen, H. W. Zhang and X. K. Wang, Removal behaviors and mechanisms of hexavalent chromium from aqueous solution by cephalosporin residue and derived chars, Bioresour. Technol., 2017, 238, 484-491.
43 S. X. Liu, X. Chen, X. Y. Chen, Z. F. Liu and H. L. Wang, Activated carbon with excellent chromium(vi) adsorption performance prepared by acid-base surface modification, J. Hazard. Mater., 2007, 141, 315-319.

44 G. T. Zhu, X. J. Xing, J. Q. Wang and X. W. Zhang, Effect of acid and hydrothermal treatments on the dye adsorption properties of biomass-derived activated carbon, J. Mater. Sci., 2017, 52, 7664-7676.

45 Z. G. Liu and F. S. Zhang, Removal of lead from water using biochars prepared from hydrothermal liquefaction of biomass, J. Hazard. Mater., 2009, 167, 933-939. 